\title{
Undifferentiated Sarcoma, Not Otherwise Specified
}

National Cancer Institute

\section{Source}

National Cancer Institute. Undifferentiated Sarcoma, Not Otherwise Specified. NCI

Thesaurus. Code C121804.

An undifferentiated soft tissue sarcoma which cannot be further characterized. 